\title{
Avaliação de sobrecarga mecânica na coluna lombar de carregadores de produtos hortícolas: um estudo de caso
}

José Wendel dos Santos wendel@email.com

niversidade Federal de Sergipe (UFS), São Cristóvão, Sergipe, Brasil

\section{José Welton dos Santos} weltonsts15@gmail.com

Instituto Federal de Educação, Ciência e Tecnologia de Sergipe (IFS), Aracaju, Sergipe, Brasil

Luciano Fernandes Monteiro lucianofm2007@gmail.com Universidade Federal de Sergipe (UFS) São Cristóvão, Sergipe, Brasil

\begin{abstract}
RESUMO
O objetivo deste estudo foi avaliar a existência de sobrecarga mecânica na coluna lombar de carregadores de produtos hortícolas da Central de Abastecimento do Estado de Sergipe (CEASA/SE). Para tanto, foram coletados dados de variáveis associadas ao manuseio de cargas executadas por 60 carregadores e das características da lombalgia autorreferida. A intensidade da dor e o grau de incapacidade funcional foram avaliados pela Escala Visual Numérica (EVN) e Questionário de Incapacidade de Roland-Morris (QIRM), respectivamente. A sobrecarga mecânica na coluna lombar e o risco de desenvolvimento de lombalgia foram avaliados pela Equação de Levantamento do NIOSH (ELN) e pelo Programa Tridimensional de Predição de Postura e de Força Estática (3DSSPP). Os resultados demonstraram que o nível de intensidade da dor e o grau de incapacidade funcional dos carregadores foram significativos. Além disso, foi observado que os carregadores manusearam cargas com peso acima do recomendado que, consequentemente, ocasionaram forças de compressão estática multidirecionais sobre os discos L4/L5 e L5/S1 da coluna vertebral superiores aos limites estipulados na literatura. Dessa forma, as fortes correlações entre as variáveis analisadas demonstraram que a tarefa desenvolvida se mostra danosa à saúde dos carregadores.
\end{abstract}

PALAVRAS-CHAVE: Sobrecarga mecânica. Manuseio de cargas. Carregador. Lombalgia. 


\section{INTRODUÇÃO}

Impulsionadas pelo desenvolvimento do país e de suas fronteiras agroindustriais, as Centrais Estaduais de Abastecimento (CEASA) foram criadas na década de $70 \mathrm{com}$ o intuito de escoar a produção agrícola de forma rápida e ordenada nos centros urbanos. De acordo com dados do Sistema de Informação de Mercados de Abastecimento do Brasil (SIMAB, 2019) das 62 CEASAs brasileiras, 53,2\% estão localizadas na região Sudeste, $19,4 \%$ no Nordeste, $16,1 \%$ no Sul, $6,5 \%$ no Centro-Oeste e $4,8 \%$ no Norte. No ano de 2018 , as CEASAs movimentaram, aproximadamente, 16,4 milhões de toneladas, o que correspondeu ao faturamento superior a $\mathrm{R} \$ 34,1$ bilhões.

Entre as diversas formas de trabalho que ocorrem nas CEASAs, destaca-se o trabalho dos carregadores, responsáveis pelo carregamento e descarregamento das mercadorias dos caminhões. No entanto, observa-se que grande parte do contingente de trabalhadores labora de forma ainda rudimentar: levantando e transportando cargas de diferentes pesos, formas e tamanhos manualmente.

Terminologicamente, o manuseio de cargas designa todo transporte no qual o peso da carga é suportado exclusivamente por um único trabalhador, compreendendo o levantamento e a deposição final da carga (BRASIL, 1990). Esse tipo de atividade exige destreza dos carregadores, pois além de requerer força física para sustentar as cargas, demanda a manutenção do equilíbrio do peso em trajetos compostos por áreas com mau estado de conservação, e condicionados ao intenso tráfego de veículos e de pessoas no local. Assim, quando realizado de forma continuada e em condições inadequadas, ocasiona forças de compressão sobre os discos intervertebrais da coluna lombar dos trabalhadores que contribuem para o desenvolvimento de lombalgias (DREISCHARF et al., 2016; MONTEIRO et al., 2017; VIOLANTE; MATTIOLI; BONFIGLIOLI, 2015).

De acordo com Santos e Monteiro (2018), as lombalgias são um problema de saúde pública em todo o mundo, em virtude de sua elevada taxa de ocorrência e predominância, custos de tratamento e o grau relativamente reduzido de êxito em sua prevenção. Essa alta incidência de lombalgias em trabalhadores que manuseiam cargas tem levado as empresas e a comunidade científica a operacionalizarem estudos no intuito de identificar os principais fatores de risco, para assim, serem implementadas medidas de controle.

No contexto brasileiro, embora não existam estudos epidemiológicos que investiguem o quadro real de como os carregadores adoecem, os dados pecuniários disponibilizados pelo Ministério da Previdência Social (MPS) estimaram que, em 2016, 62\% dos casos de afastamento por incapacidade laboral foram decorrentes de lesões na coluna vertebral. Os dados revelaram ainda, que estes benefícios representaram um ônus financeiro de $\mathrm{R} \$ 117,3$ milhões tanto para as empresas quanto para a sociedade (MONTEIRO et al., 2016).

Nesta perspectiva, justificam-se estudos epidemiológicos em perspectiva ergonômica que buscam esclarecer a gênese da morbidade causada pela lombalgia nesta população. Dessa forma, o objetivo deste estudo foi avaliar a existência de sobrecarga mecânica na coluna lombar de carregadores de produtos hortícolas da Central de Abastecimento do Estado de Sergipe (CEASA/SE). 


\section{METODOLOGIA DA PESQUISA}

\section{TIPO DE ESTUDO E CASUÍSTICA}

Este estudo transversal foi conduzido na Central de Abastecimento do Estado de Sergipe (CEASA/SE), localizada na cidade de Aracaju. O espaço foi construído em 1973 para receber e redistribuir a produção de hortifrutigranjeiros no estado.

Participaram deste estudo 60 carregadores de produtos hortícolas, todos do sexo masculino com idade média de $38 \pm 9$ anos, altura média de 1,75 $\pm 0,05 \mathrm{~m}$, peso médio de $77,3 \pm 6,1 \mathrm{~kg}$, IMC médio de $25,2 \pm 2,3 \mathrm{~kg} \cdot \mathrm{m}^{-2}$. O tempo médio de serviços prestados por esses profissionais foi de $8 \pm 5$ anos e jornada de trabalho média de $7 \pm 1$ horas.

\section{PROCEDIMENTOS DE COLETA DE DADOS}

A coleta de dados ocorreu no segundo semestre de 2018 e foi sistematizada em três etapas. Na primeira etapa, foram conduzidas visitas in loco para conhecer as instalações físicas do entreposto, a logística de preparação e distribuição das mercadorias, selecionar os voluntários para compor o Grupo Homogêneo de Exposição (GHE) e definir a agenda de pesquisa de campo.

Na segunda etapa, foram coletadas as variáveis da tarefa de levantamento e transporte de cargas: distância horizontal $(H)$, distância vertical $(V)$, distância vertical percorrida (D), assimetria da carga $(A)$, frequência de levantamentos (F) e pega da carga (P). Utilizou-se, para tanto, a balança digital Avanutri $^{\circledR}$, goniômetro Trident $^{\circledast}$, trena métrica Starrett ${ }^{\circledast}$ e cronômetro digital Vollo ${ }^{\circledast}$. Além disso, foram realizadas as observações sistêmicas da biomecânica utilizada pelos carregadores durante a execução das tarefas. Esses dados foram documentados e registrados por meio de fotos e filmagens utilizando duas câmeras digitais Samsung ${ }^{\circledR}$.

$\mathrm{Na}$ terceira etapa, os dados relacionados às características da lombalgia autorreferida pelos carregadores foram coletados. A Escala Visual Numérica (EVN) foi utilizada para medir a intensidade da lombalgia, recorrendo-se a uma linha horizontal graduada de 0 a $10 \mathrm{com}$ os extremos demarcados com os descritores "sem dor" e "dor severa". Assim, cada carregador localizou espacialmente o quanto de dor estava sentindo antes e após a execução da tarefa. Os valores de 0 a 3 foram classificados como "dor leve", de 4 a 7 como "dor moderada" e acima de 8 como "dor forte" (HJERMSTAD et al., 2011).

O Questionário de Incapacidade de Roland-Morris (QIRM) foi utilizado para medir o grau de incapacidade funcional do carregador como resultado da lombalgia. Nessa avaliação, o pesquisador realizou a leitura de 24 sentenças que descreviam limitações funcionais comumente relacionadas à lombalgia. As respostas dadas pelos carregadores eram dicotômicas e o resultado final correspondeu à soma das respostas positivas. Dessa maneira, o resultado pode, portanto, variar entre 0 (sem incapacidade funcional) e 24 (incapacidade funcional severa), sendo que os indivíduos que obtiveram escores superiores a 14 foram diagnosticados com incapacidade funcional significativa (ROLAND; MORRIS, 1983). 


\section{PROCEDIMENTOS DE ANÁLISE DOS DADOS}

$\mathrm{Na}$ análise dos dados, inicialmente, utilizou-se a Equação de Levantamento do NIOSH (ELN), proposta pelo National Institute for Occupational Safety and Health (NIOSH), para avaliar e determinar o Limite de Peso Recomendado (LPR) para a tarefa realizada pelos carregadores. O LPR foi obtido pela Equação (1).

$$
\mathrm{LPR}=\mathrm{CC} \times \mathrm{MH} \times \mathrm{MV} \times \mathrm{MD} \times \mathrm{MA} \times \mathrm{MF} \times \mathrm{MP}
$$

Em que:

CC Constante de carga. Corresponde ao peso ideal de levantamento: $23 \mathrm{~kg}$.

$\mathrm{MH}$ Multiplicador de distância horizontal da pega ao ponto médio entre os tornozelos, dado por $(25 / \mathrm{H})$.

Multiplicador de altura vertical das mãos em relação ao solo no início

MV da elevação, dado por (1 - $(0,0038 \times[V-75])$ para alturas acima de 75 $\mathrm{cm}$ e $(1-(-0,003 \times[\mathrm{V}-75])$ para alturas até $75 \mathrm{~cm}$. destino, dado por $(0,82+(4.5 / D))$.

MA Multiplicador de assimetria da carga, dado por $(1-(0.0032 \times A))$

MF Multiplicador de frequência de levantamentos por minuto, obtido na Tabela 1.

MP Multiplicador de qualidade da pega, obtido na Tabela 2.

O MF foi obtido a partir do número de $F, V$ e da duração da tarefa, conforme apresentado na Tabela 1.

Tabela 1 - Valores de MF

\begin{tabular}{|c|c|c|c|c|c|c|}
\hline \multirow{3}{*}{$\begin{array}{c}\text { F } \\
\text { (levs./min.) }\end{array}$} & \multicolumn{6}{|c|}{ Duração do trabalho (h/dia) } \\
\hline & \multicolumn{2}{|c|}{$\leq 1 \mathrm{~h}$} & \multicolumn{2}{|c|}{$\leq 2 h$} & \multicolumn{2}{|c|}{$\leq 8 h$} \\
\hline & $V<75 \mathrm{~cm}$ & $V \geq 75 \mathrm{~cm}$ & $V<75 \mathrm{~cm}$ & $V \geq 75 \mathrm{~cm}$ & $V<75 \mathrm{~cm}$ & $V \geq 75 \mathrm{~cm}$ \\
\hline 0,2 & 1,00 & 1,00 & 0,95 & 0,95 & 0,85 & 0,85 \\
\hline 0,5 & 0,97 & 0,97 & 0,92 & 0,92 & 0,81 & 0,81 \\
\hline 1 & 0,94 & 0,94 & 0,88 & 0,88 & 0,75 & 0,75 \\
\hline 2 & 0,91 & 0,91 & 0,84 & 0,84 & 0,65 & 0,65 \\
\hline 3 & 0,88 & 0,88 & 0,79 & 0,79 & 0,55 & 0,55 \\
\hline 4 & 0,84 & 0,84 & 0,72 & 0,72 & 0,45 & 0,45 \\
\hline 5 & 0,80 & 0,80 & 0,60 & 0,60 & 0,35 & 0,35 \\
\hline
\end{tabular}

Fonte: Waters et al. (1993)

O MP foi obtido em função de $\mathrm{P}$, conforme disposto na Tabela 2. Assim, P foi considerado bom quando a carga possuía alça ou ponto de apoio que possibilitasse o manejo de preensão palmar; razoável, quando permitiu manejos com as mãos flexionadas em torno de $90^{\circ}$; e ruim, quando a carga não possuía ponto de apoio.

Tabela 2 - Valores de MP

\begin{tabular}{ccc}
$P$ & & $M P$ \\
Bom & $V \leq 75 \mathrm{~cm}$ & $V \geq 75 \mathrm{~cm}$ \\
Razoável & 1,00 & 1,00 \\
Ruim & 0,95 & 1,00 \\
\hline
\end{tabular}

Fonte: Waters et al. (1993) 
A partir do LPR, calculou-se o Índice de Levantamento (IL), dado pela divisão da carga real levantada pelo LPR. Segundo Waters et al. (1993), quando o valor do IL se encontra no intervalo de 0 a 1 , a sobrecarga mecânica na coluna lombar do trabalhador é pequena e a chance do desenvolvimento de lombalgia será mínima. Valores entre 1,1 e 2,9 aumenta-se o risco. Valor igual ou superior a 3 indica grande probabilidade de lesões na coluna vertebral do trabalhador.

Em seguida, o Programa de Predição de Postura e de Força Estática 3D (3DSSPP), desenvolvido pelo Centro de Ergonomia da Universidade de Michigan, foi utilizado para mensurar as forças de compressão multidirecionais sobre os discos da coluna lombar dos carregadores. Os modelos biomecânicos foram construídos a partir de dados antropométricos, das variáveis da tarefa e das posturas típicas adotadas no ciclo de trabalho. A representação gráfica do modelo biomecânico baseado na postura do carregador, bem como das bases ortonormais nos planos anatômicos $\mathrm{x}$, y e z pode ser visualizada na Figura 1 .

Figura 1 - Representação do modelo biomecânico do carregador

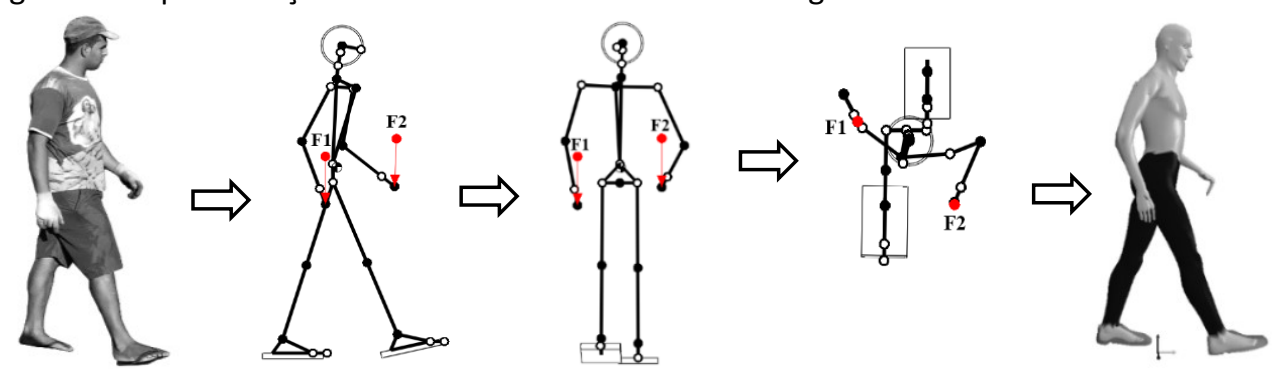

Fonte: Elaborada pelo autor no 3DSSPP ${ }^{\circledR}$ (2018)

Por fim, as informações obtidas foram armazenadas em banco de dados elaborado no programa Statistical Package for the Social Scienses (SPSS 24) para Windows ${ }^{\circledR}$, no qual foram conduzidas as análises estatísticas. Na análise univariada dos dados, verificou-se a distribuição de frequência, medidas de posição e de dispersão dos dados. Além disso, utilizaram-se os testes não paramétricos de Kruskal-Wallis, Mann-Whitney e Wilcoxon para comparação entre grupos, ao nível de significância de $p<0,05$.

$\mathrm{Na}$ análise bivariada dos dados, o coeficiente de correlação linear de Pearson $(r)$ foi utilizado para verificar a força e a direção da relação entre as variáveis do estudo. Considerou-se correlação fraca quando $0<r<0,4$; moderada quando 0,4 $<r<0,7$ e forte quando $0,7<r<1,0$, conforme sugerido por Schober, Boer e Schwarte (2018). Ademais, utilizou-se o coeficiente de determinação $\left(r^{2}\right)$ para verificar, em porcentagem, a influência de uma variável sobre a outra (MALHOTRA, 2005).

\section{ASPECTOS ÉTICOS}

Este estudo foi aprovado pelo Comitê de Ética em Pesquisa (CEP) da Fundação Universidade Federal de Sergipe (FUFS), sob parecer no 2203949. Todos os carregadores assinaram o Termo de Consentimento Livre e Esclarecido (TCLE) e foram instruídos sobre a natureza voluntária do estudo, os procedimentos adotados no transcorrer da pesquisa de campo e a confidencialidade da sua identidade e condição funcional. 


\section{CARACTERIZAÇÃO DO LOCAL DE TRABALHO}

Considerado o principal entreposto do Estado de Sergipe, a CEASA/SE possui área total de 34 mil metros quadrados, sendo 10 mil de área construída, onde estão instalados o Mercado Permanente (MP), Mercado Não Permanente (MNP) e Mercado do Produtor Rural (MPR), além do prédio administrativo e lojas prestadoras de serviços. O MP é constituído por um pavilhão fechado subdividido em pontos de comércio individualizados. O MNP refere-se ao pavilhão aberto com marcas no solo que delimitam o local de comercialização dos produtos hortifrutigranjeiros no espaço. O MPR engloba galpões fechados destinados prioritariamente ao produtor rural para comercialização de sua produção. $\mathrm{Na}$ Figura 2 pode-se verificar a localização espacial desses mercados dentro da CEASA/SE.

Figura 2 - Localização espacial dos mercados dentro da CEASA/SE

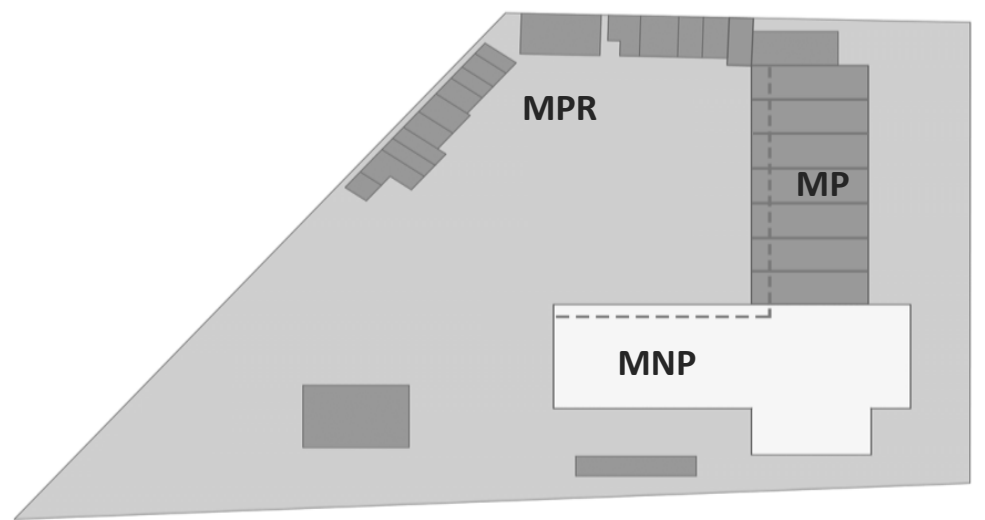

Fonte: Autoria própria (2018)

$\mathrm{Na}$ CEASA/SE, a comercialização dos produtos é realizada por agentes de mercado detentores de concessão para a utilização do espaço. Segundo a Associação dos Usuários da Ceasa de Aracaju (ASSUCEAJU, 2018), as firmas constituídas pelos 249 agentes de mercado comercializam os mais variados produtos, que são subdivididos em hortaliças, legumes, frutas, verduras, cereais e outros gêneros alimentícios. Essas operações ocorrem de segunda a sexta-feira das 3 às $18 \mathrm{~h}$ e aos sábados das 3 às $16 \mathrm{~h}$, por dois principais canais de venda: atacado e varejo. As empresas comerciais e industriais são os principais clientes dos agentes de mercado.

Os produtos comercializados no entreposto são procedentes de diversos municípios do estado de Sergipe e importados de outras regiões do país. De acordo com a ASSUCEAJU (2018), os municípios de Itabaiana, Malhador, Neópolis, Pacatuba, Santana do São Francisco, Telha, Propriá, Boquim, Moita Bonita, Ribeirópolis e Campo do Brito se destacam como principais produtores agrícolas no estado, e respondem por $60 \%$ do volume de produtos comercializados. Os produtos importados advêm, geralmente, dos estados da Bahia, Pernambuco, Alagoas, Minas Gerais, Distrito Federal, Paraná, São Paulo, Rio Grande do Sul e Santa Catarina. 
A CEASA/SE recebe, em média, 186 veículos cargueiros diariamente. O volume de cargas movimentadas no entreposto expandiu muito desde a sua criação. Em 1973, a média diária era de 10 mil toneladas, ampliando para 25 mil em 1988 e 60 mil em 2017 (ASSUCEAJU, 2018). Para atender a demanda, as firmas intensificaram a contratação de carregadores, e assim, ampliar a sua capacidade logística. Assim como nas demais centrais brasileiras, os carregadores da CEASA/SE exercem papel indispensável nas operações de compra e venda de mercadorias.

\section{CARACTERIZAÇÃO DO PROCESSO DE TRABALHO}

O trabalho realizado pelos carregadores consistiu no carregamento e descarregamento de caminhões cargueiros em diferentes pontos das instalações da CEASA/SE. O modo de operação seguiu o mesmo padrão dos demais entrepostos brasileiros, cujos processos ainda não foram automatizados: manusear cargas de diferentes tipos, dimensões e pesos utilizando como principal instrumento de trabalho o seu próprio corpo.

Os sessenta veículos carregados/descarregados durante a pesquisa de campo eram, em sua maioria, não articulados com capacidade de carga de 16 a 23 toneladas. Sobre o assoalho das carrocerias encontravam-se dois tipos de embalagens: caixas de madeira ou de plástico e sacarias. As caixas possuíam formato retangular com abertura para nas laterais. As sacarias eram confeccionadas em diferentes tipos de materiais e possuíam capacidade para embalar até $60 \mathrm{~kg}$ de produtos hortícolas.

Operacionalmente, os carregadores manusearam, em média, $233 \pm 71$ unidades de cargas com peso médio de $44,3 \pm 8,0 \mathrm{~kg}$. A distância média percorrida pelos carregadores com a carga foi de $38,7 \pm 21,6$ metros, e todo o processo de carregamento/descarregamento dos caminhões teve duração média de $51,9 \pm 13,4$ minutos.

O posto de trabalho teve influência direta no manuseio das mercadorias. As operações eram realizadas ora em plataforma, ora com o carregador posicionado no solo. A plataforma conjugada, em formato $L$, estava instalada nas áreas de expedição do MP e MNP, as quais mediam, respectivamente, 86,1 e $57,9 \mathrm{~m}$ de comprimento e 1,42 $\mathrm{m}$ de altura. Essas dimensões permitiram o nivelamento da sua base com as carrocerias dos caminhões, facilitando, portanto, na redução do trajeto percorrido pelo carregador. A Figura 3 ilustra o processo de descarregamento de mercadorias na plataforma do MP.

Figura 3 - Processo de descarregamento de mercadorias na plataforma do MP

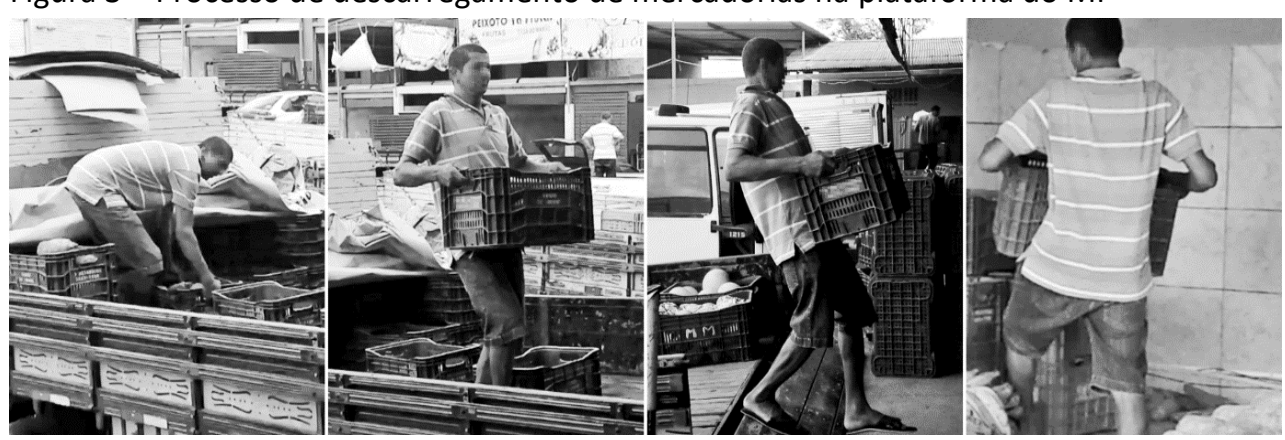

Fonte: Dados da pesquisa (2018) 
O MPR não dispunha de área de expedição para o carregamento e descarregamento das mercadorias, assim, a operação foi realizada com o carregador posicionado no solo. A indisponibilidade de vaga na plataforma do MP ou MNP também contribuiu para sua execução nessa condição, conforme ilustrado nas Figuras 4 e 5.

Figura 4 - Processo de carregamento de mercadorias a partir do solo no MPR
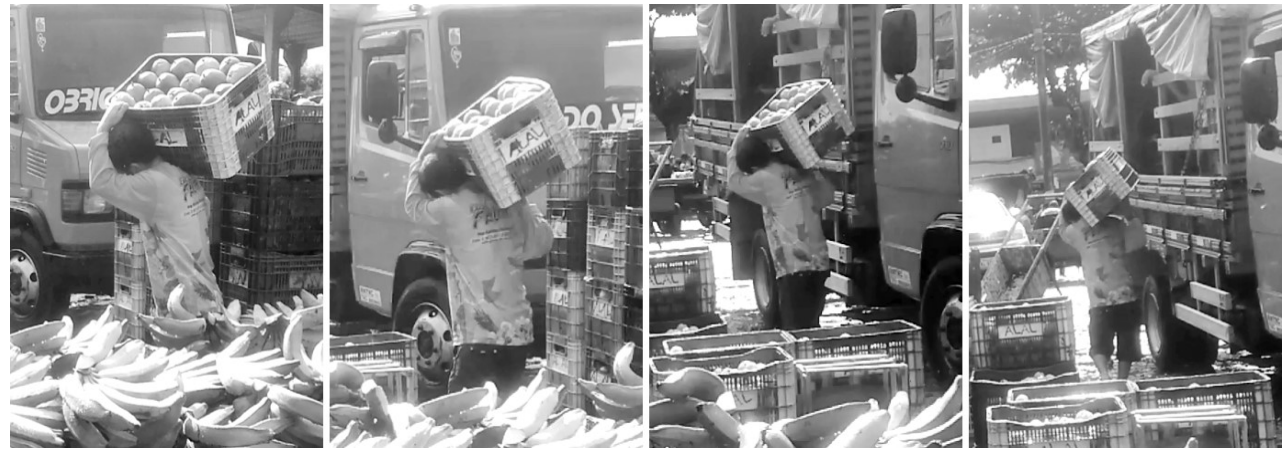

Fonte: Dados da pesquisa (2018)

Figura 4 - Processo de carregamento de mercadorias a partir do solo no MNP
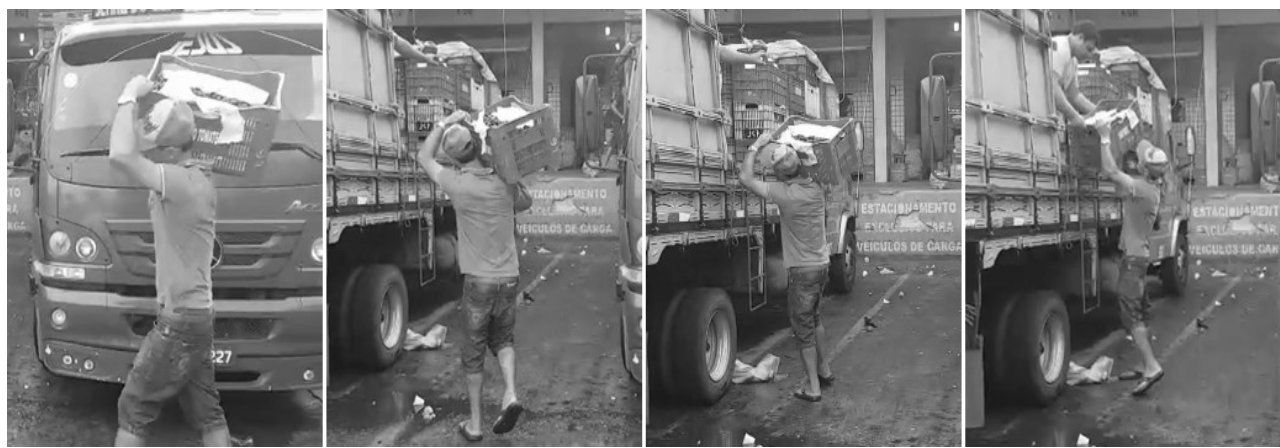

Fonte: Dados da pesquisa (2018)

\section{CARACTERISTICAS DA LOMBALGIA AUTORREFERIDA}

$\mathrm{Na}$ entrevista, os carregadores foram questionados quanto à presença ou ausência de manifestações lombálgicas. Na ocasião, 30 carregadores reportaram dores lombares, revelando uma prevalência de 50\%. Esses carregadores atribuíram o quadro álgico ao trabalho realizado e afirmaram ter tido pelo menos um episódio de lombalgia nos últimos três meses anteriores à entrevista. Desses carregadores, 83,5\% foram acometidos por lombalgia nos últimos sete dias. Nessas ocasiões, $86,7 \%$ dos carregadores não procuraram assistência médica, porém $53,3 \%$ se automedicaram ou fizeram uso de terapias alternativas para o controle das crises, como por exemplo, a ingestão de chás medicinais.

No que se refere à frequência das lombalgias, foi observado que $50 \%$ dos carregadores alegaram ter episódios semanalmente, enquanto $33,3 \%$ diariamente, $10 \%$ quinzenalmente e $6,7 \%$ mensalmente. Em $66,7 \%$ dos casos, o tempo de recuperação foi inferior a três dias e em $33,3 \%$ foi superior. Durante as crises lombálgicas, $60 \%$ dos carregadores afirmaram ter faltado ao trabalho e apenas $6,7 \%$ reduziram o ritmo de trabalho. Os dados relacionados às 
características da lombalgia autorreferida pelos carregadores estão organizados na Tabela 3.

Tabela 3 - Características da lombalgia autorreferida pelos carregadores $(n=30)$

$\begin{array}{lcc}\text { Característica } & \text { Total } & \% \\ \text { Episódios de lombalgia nos últimos: } & 30 & 100 \\ \quad 3 \text { meses } & 25 & 83,3 \\ \quad 7 \text { dias } & & \\ \text { Procura por assistência médica } & 26 & 86,7 \\ \quad \text { Não } & 4 & 13,3 \\ \quad \text { Sim } & 16 & 53,3 \\ \text { Uso de medicamentos ou terapias alternativas } & 14 & 46,7 \\ \quad \text { Sim } & & \\ \quad \text { Não } & 15 & 50 \\ \text { Frequência dos episódios de lombalgia } & 10 & 33,3 \\ \quad \text { Semanal } & 3 & 10 \\ \quad \text { Diária } & 2 & 6,7 \\ \text { Quinzenal } & & \\ \quad \text { Mensal } & 20 & 66,7 \\ \text { Tempo de recuperação } & 10 & 33,3 \\ \quad \text { > dias } & & \\ \quad \text { < dias } & & 60 \\ \text { Falta ao trabalho } & 18 & 40 \\ \quad \text { Sim } & 12 & \\ \quad \text { Não } & & 93,3 \\ \text { Redução do ritmo de trabalho } & 28 & 6,7 \\ \quad \text { Não } & 2 & \\ \text { Sim }\end{array}$

Fonte: Dados da pesquisa (2018)

O nível de intensidade da lombalgia relatada na EVN pode ser observado na Figura 6. Na avaliação inicial, a intensidade média foi de $4,3 \pm 0,9$ pontos, com variação entre intensidade leve (3) e moderada (6). Na reavaliação, a intensidade média foi 7,3 $\pm 1,1$ pontos, e variou entre intensidade moderada (6) e forte (9). Nenhum carregador reportou ausência de dor (0) ou dor severa (10).

Figura 6 - Intensidade da lombalgia autorreferida pelos casos $(n=30)$

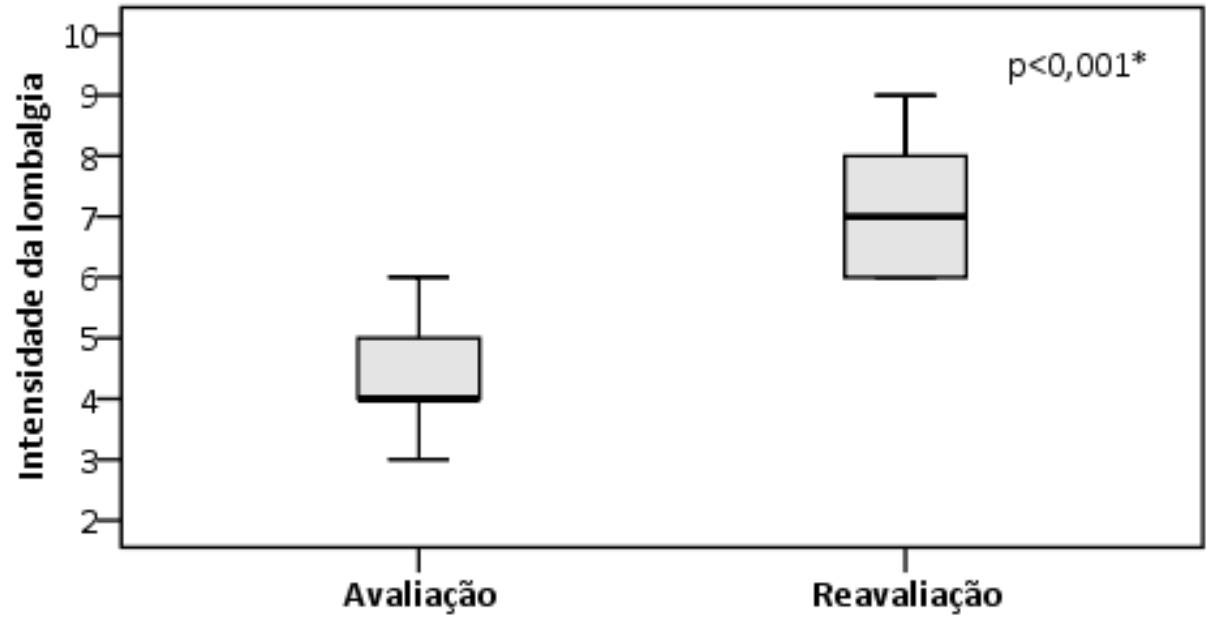

Fonte: Autoria própria (2018)

Nota: *Teste de Wilcoxon. 
Houve diferenças estatisticamente significativas entre os níveis de intensidade da dor nas duas ocasiões de avaliação $(p<0,001)$, bem como forte correlação estatística entre as duas medidas $(r=0,87 ; p<0,001)$, evidenciando a influência do trabalho realizado na intensidade da dor experimentada pelos carregadores.

Destaca-se ainda que, houve diferenças estatisticamente significativas entre os carregadores no que se refere à procura por assistência média $(p<0,001)$ e uso de terapia alternativas $(p<0,05)$ quando acometidos pela lombalgia, pois esses indivíduos foram os que apresentaram maior intensidade de dor nas duas ocasiões de avaliação. Fato semelhante foi observado nos carregadores com episódios diários de lombalgia $(p<0,001)$ e quando o tempo de recuperação foi superior a três dias $(p<0,01)$.

Diante desse quadro, recorreu-se ao QIRM para verificar o grau de incapacidade funcional dos carregadores resultante das crises lombálgicas. Nessa avaliação, observou-se que a média das respostas positivas dadas pelos carregadores referentes às assertivas do QIRM foi de $13,5 \pm 1,9$ pontos. Nenhum carregador apresentou ausência de incapacidade funcional (0) ou incapacidade funcional severa (24 pontos), conforme evidenciado na Figura 7. 0 grau de incapacidade funcional dos carregadores variou entre 9 e 17 pontos. Os escores obtidos por $46,7 \%$ dos carregadores não atingiram o limiar de incapacidade funcional (14 pontos), enquanto $53,3 \%$ foram enquadrados como portador de incapacidade funcional significativa.

Figura 7 - Grau de incapacidade funcional dos casos $(n=30)$

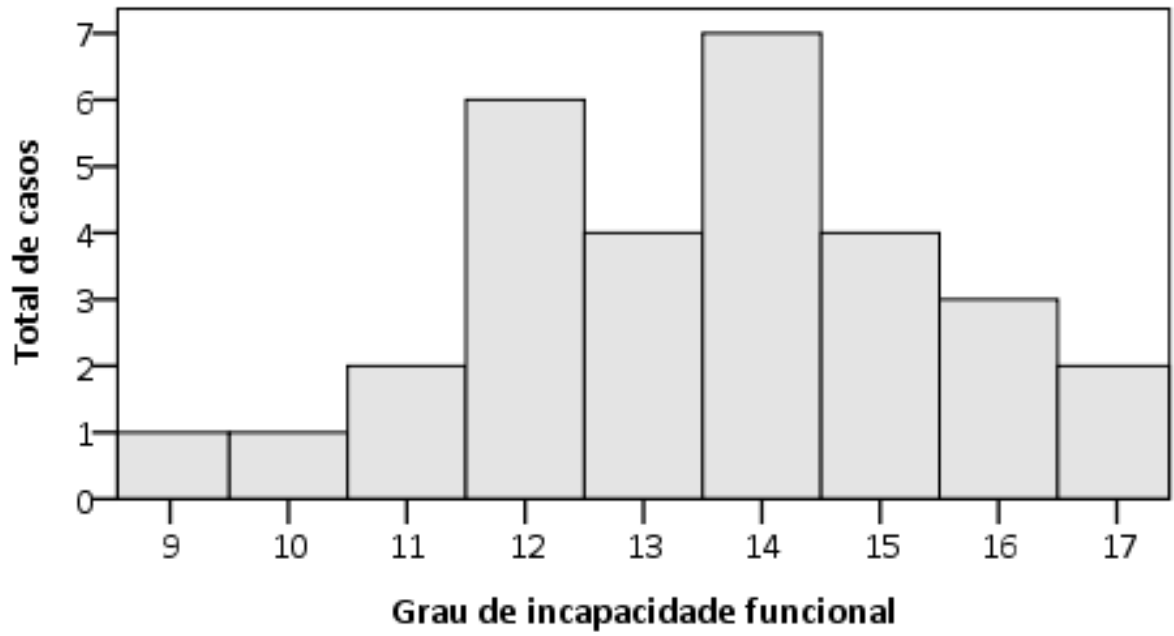

Fonte: Autoria própria (2018)

A esse respeito, constatou-se forte correlação estatística entre o nível de intensidade da lombalgia e o grau de incapacidade funcional dos carregadores ( $r$ $=0,89 ; p<0,001)$. Em termos percentuais, o $r^{2}$ indicou que 79,2\% do grau de incapacidade funcional dos carregadores podem ser explicados pelos níveis de intensidade da dor. Ou seja, quanto maior foi a sua intensidade, mais incapacitante se mostrou a lombalgia.

Além disso, foram constatadas diferenças estatisticamente significativas entre o grau de incapacidade funcional dos carregadores em relação à frequência das crises $(p<0,05)$, pois os carregadores que relataram crises diárias mostraram-se com maior grau de limitação funcional. De modo semelhante 
ocorreu nos carregadores com maior tempo de recuperação $(p<0,05)$, principalmente quando esse período foi superior a quatro dias. Os carregadores diferiram entre si, também, quanto à falta ao trabalho $(p<0,001)$ e quanto à redução do ritmo de trabalho $(p<0,001)$. Nesse cenário, apesar do número reduzido de carregadores que declararam faltar ao trabalho ou reduzir a carga de trabalho, os que o fizeram apresentaram grau de incapacidade funcional superior aos demais. Esses aspectos podem ser verificados na Figura 8.

Figura 8 - Comparação entre o grau de incapacidade funcional dos carregadores $(n=30)$
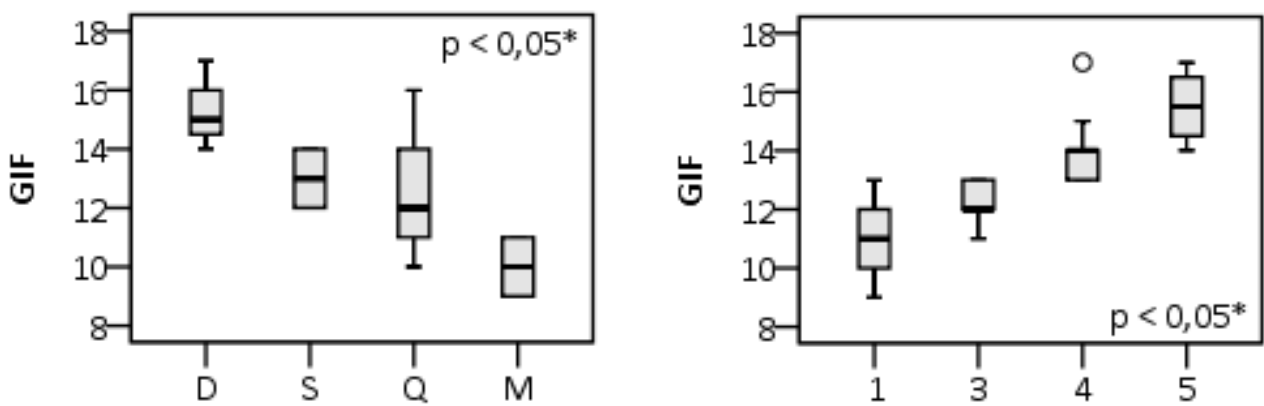

Frequência das crises
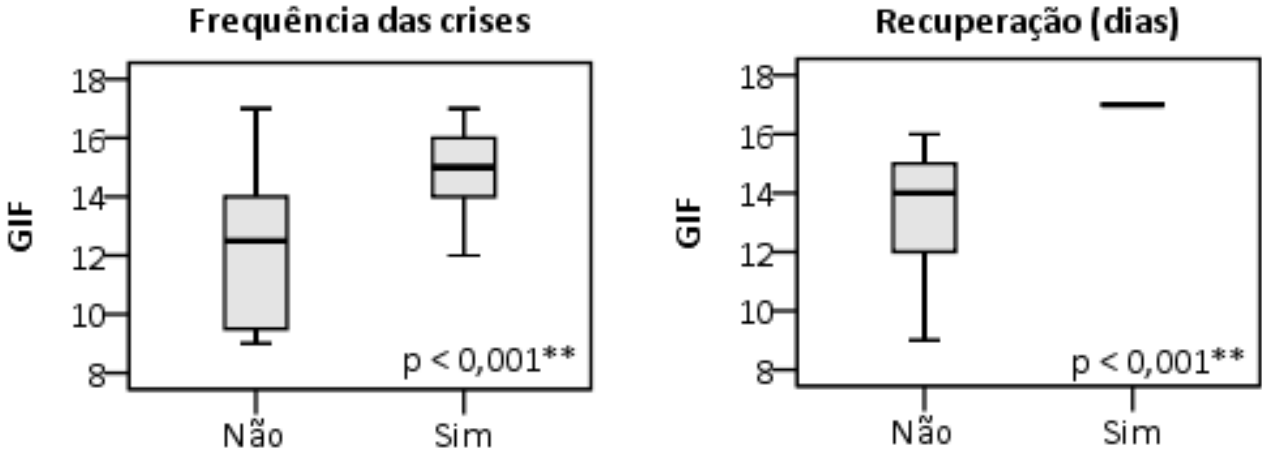

Falta ao trabalho

Redução do trabalho

Fonte: Autoria própria (2018)

Legenda: $\mathrm{D}=$ Diária; $\mathrm{S}=$ Semanal; $\mathrm{Q}=$ Quinzenal; $\mathrm{M}=$ Mensal; $\mathrm{GIF}=$ grau de incapacidade funcional.

Nota: *Teste de Kruskal-Wallis; **Teste de Mann-Whitney.

O alto nível de intensidade da dor e o grau de incapacidade funcional sugere a existência de sobrecarga mecânica na coluna lombar dos carregadores. Acredita-se que, por não possuírem vínculo empregatício ou filiação com sindicatos da categoria, a falta de informação, a necessidade de responder às exigências do trabalho e outras contingências estimulam esses profissionais a suportarem as crises álgicas e a continuarem executando o trabalho como se nada estivesse ocorrendo. O retorno financeiro rápido, seja para sustentar a si próprio ou à sua família tem forte influência nessas decisões.

De acordo com Silva (2009), essa busca incessante da manutenção dos seus rendimentos induz o carregador a ultrapassar os limites fisiológicos que, em uma situação precária de trabalho, são atingidos de várias formas. Dessa forma, mostra-se relevante investigar a existência de sobrecarga mecânica na coluna lombar dos carregadores em decorrência das tarefas de levantamento e transporte manual de mercadorias para a implementação de medidas de controle. 


\section{AVALIAÇÃO DE SOBRECARGA MECÂNICA}

A avaliação de sobrecarga mecânica das tarefas de levantamento de mercadorias foi realizada pela ELN, em conformidade com os requisitos estabelecidos pelo NIOSH. Na Tabela 4 pode ser observado que a média do LPR e IL para a tarefa foi $8,2 \pm 2,5$ e 6,0 $\pm 2,2$, respectivamente. Esses valores foram influenciados pelas constantes de carga de $M A(0,7)$ e $M F(0,7)$, pois além da frequência de levantamentos serem significativas foram observadas assimetria nas elevações das cargas. Por outro lado, MP influenciou positivamente o LPR e IL, pois foi o único que apresentou valor ideal $(1,0)$ para a tarefa.

Tabela 4 - Multiplicadores da ELN, LPR e IL para a tarefa de levantamento $(n=60)$

\begin{tabular}{ccccccccc} 
CC & MH & MV & MD & MA & MF & MP & LPR & IL \\
23 & $0,8 \pm 0,1$ & $0,9 \pm 0,1$ & $0,9 \pm 0,0$ & $0,7 \pm 0,1$ & $0,7 \pm 0,1$ & $1,0 \pm 0,0$ & $8,2 \pm 2,5$ & $6,0 \pm 2,2$ \\
\hline
\end{tabular}

Fonte: Autoria própria (2018)

Constatou-se correlação forte e negativa entre os LPR e IL $(r=-0,897 ; p<$ $0,001)$ determinados para a tarefa, indicando que quanto menor foi o LPR, maior foi o IL. De fato, o $r^{2}$ indicou que pelo menos $80,5 \%$ dos IL obtido pode ser explicado pelos valores de seus respectivos LPR. Além disso, notou-se que todos os valores de IL obtidos pelos carregadores foram superiores a 3,0 e indicaram a existência de sobrecarga na coluna lombar e grande probabilidade de desenvolvimento de lombalgia se medidas de controle não forem implementadas.

No que se refere a modelagem biomecânica realizada no 3DSSPP ${ }^{\circledR}$, observou-se que as elevações das cargas ocasionaram forças de compressão média de $3342,4 \pm 631,5 \mathrm{~N}$. No Quadro 1 encontram-se as posturas adotadas pelos carregadores que ocasionaram os maiores picos de compressão intradiscal observados nessa etapa da tarefa.

Quadro 1 - Picos de compressão intradiscal na elevação das cargas

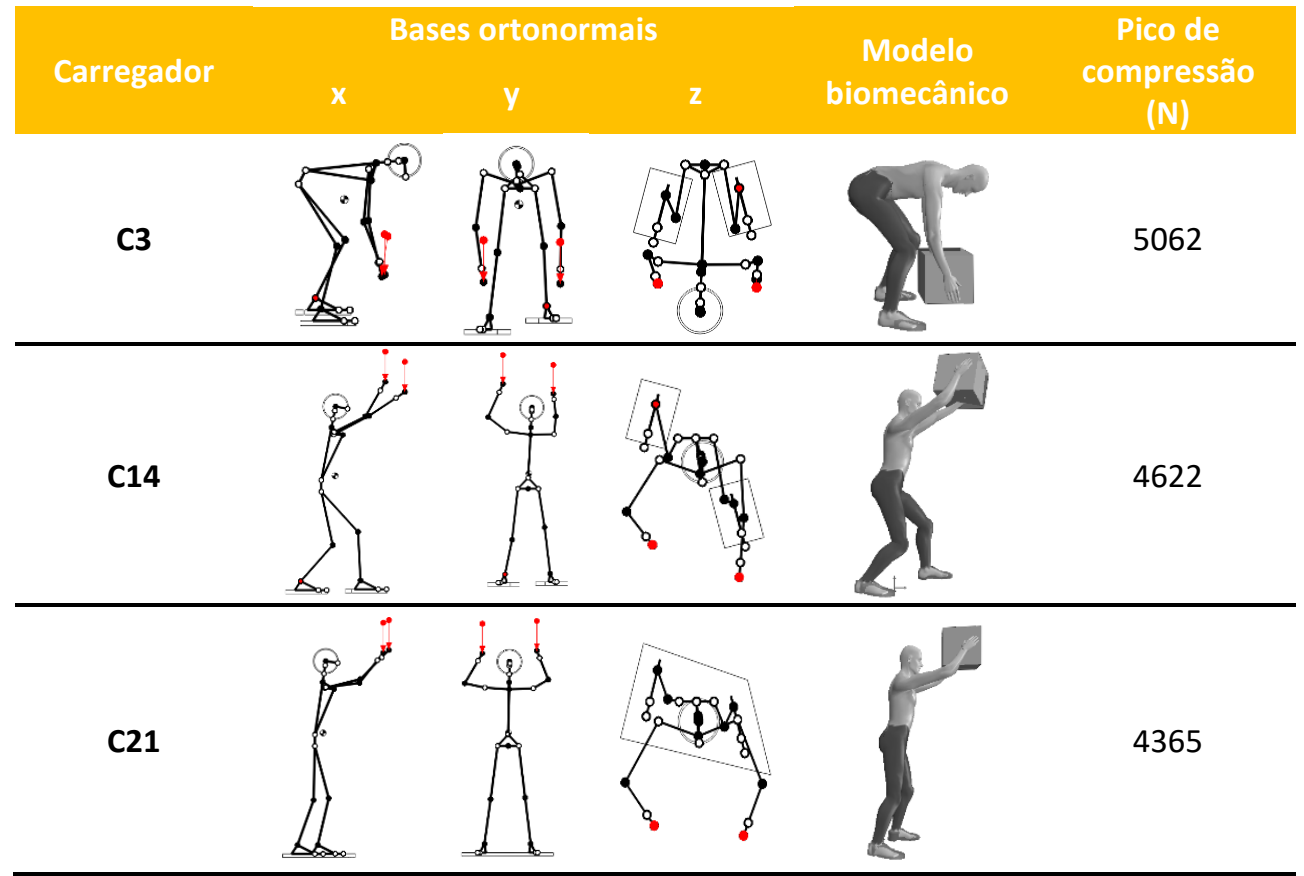

Fonte: Elaborada pelo autor no 3DSSPP ${ }^{\circledR}$ (2018) 
No transporte das cargas, a compressão média exercida na coluna lombar dos carregadores foi de 1979,2 $\pm 447,3 \mathrm{~N}$. As posturas adotadas pelos carregadores que ocasionaram os maiores picos de compressão intradiscal observados nessa etapa da MMC podem ser verificadas no Quadro 2.

Quadro 2 - Picos de compressão intradiscal no transporte das cargas

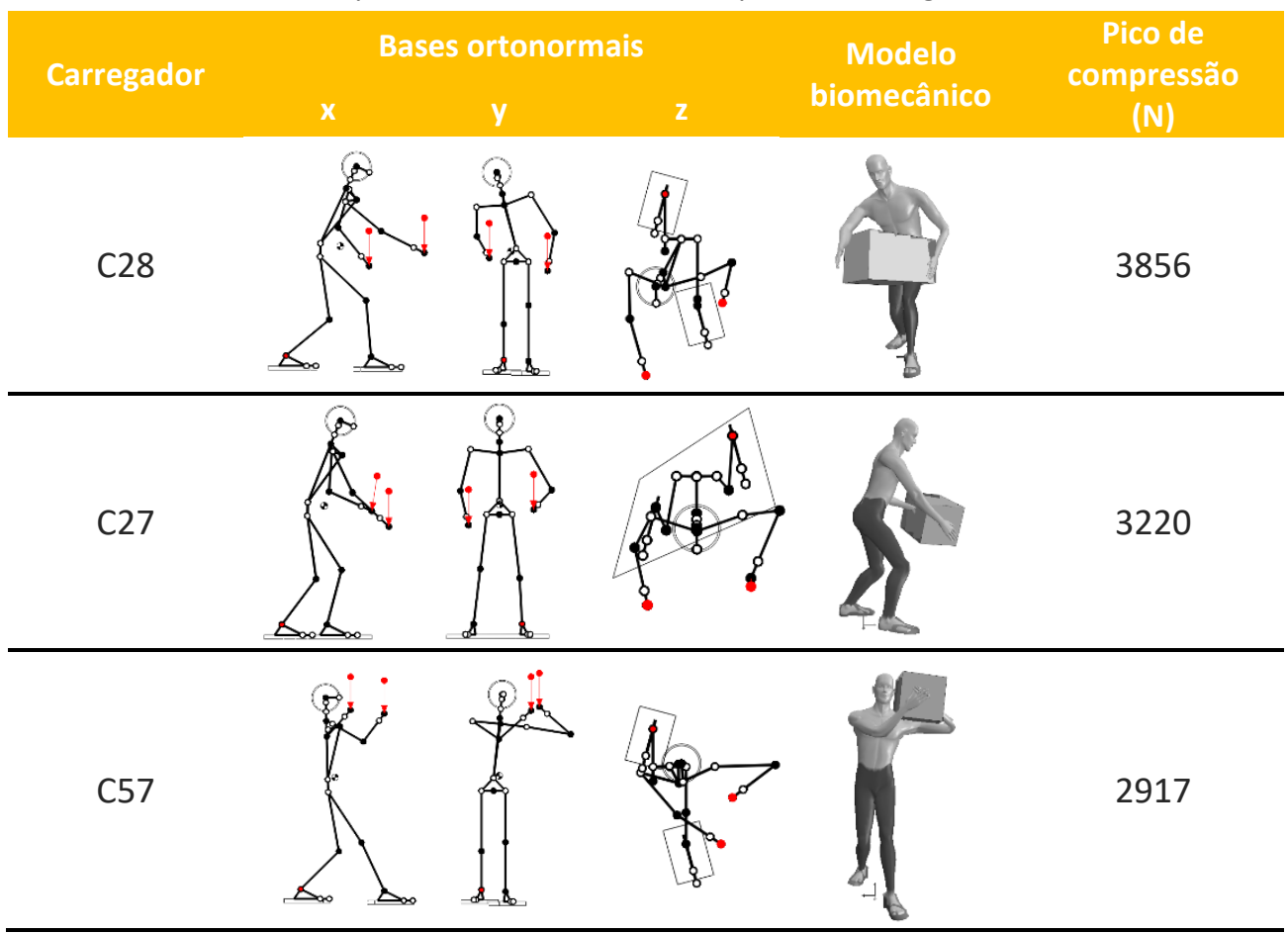

Fonte: Elaborada pelo autor no 3DSSPP ${ }^{\circledR}$ (2018)

Houve diferenças significativas entre os carregadores em relação às forças de compressão intradiscal ocasionadas pela tarefa $(p<0,001)$. Os carregadores lombálgicos estavam submetidos a maiores forças compressivas durante a execução da tarefa. Também foi verificada a existência de correlação forte e positiva entre essas forças e o peso das cargas $(r=0,91 ; p<0,001)$, IL $(r=0,86 ; p$ $<0,001)$, EVN $(r=0,84 ; p<0,001)$ e QIRM $(r=0,81 ; p<0,001)$, indicando que um aumento na compressão intradiscal contribui para o desenvolvimento de lombalgia com intensidades mais fortes e incapacitantes.

Diversos estudos associam as forças de compressão intradiscal em L1/L5 ao desenvolvimento de lombalgia (ALENCAR, 2001; BOGDUK, 2012; GORDON et al., 1991; MONTEIRO et al., 2017; PELLENZ, 2005; SANTA MARIA, 2001). Isto porque, quando uma carga é aplicada externamente à coluna lombar, as forças de compressão nos discos intervertebrais aumentam promovendo o abaulamento da fibrose do ânulo e as fibras anulares concêntricas se projetam para fora, comprimindo as raízes nervosas da coluna vertebral e ocasionando as lombalgias. Por este motivo, a força compressiva nos discos intervertebrais não deve ser superior a $3400 \mathrm{~N}$, pois quando são submetidos a compressões acima desta ordem micro traumas são gerados, que dependendo do grau de evolução, pode cronificar e resultar na morbidade e incapacidade funcional do trabalhador, conforme destacado por Adams e Hutton (1980) e Dreischarf et al. (2016).

Por sua vez, a tarefa de levantamento e transporte manual de cargas tem sido associada ao aumento da compressão intradiscal e consequente 
desencadeamento de lombalgias (MERINO, 1996; PELLENZ, 2005; SANTOS; MONTEIRO, 2017, 2018;TEIXEIRA; OKIMOTO; GONTIJO, 2011). Nesse sentido, recomenda-se que ao manusear as mercadorias, o carregador considere o peso e a posição da carga em relação ao eixo do corpo. Segundo Másculo e Vidal (2013), no transporte manual de cargas, o trabalhador deve observar o plano de levantamento, manter a carga próxima ao corpo, evitar a rotação, extensão e flexão lateral do tronco e, durante a elevação flexionar os joelhos. A eficácia dessas recomendações foi relatada nos estudos de Waters et al. (1993) e NIOSH (2015), os quais constataram a diminuição da distância corpo-carga como fator redutor da sobrecarga na coluna lombar.

\section{CONSIDERAÇÕES FINAIS}

O objetivo deste estudo foi avaliar a existência de sobrecarga mecânica na coluna lombar de carregadores de produtos hortícolas da CEASA/SE. Nesta análise, observou-se que todos os carregadores que relataram dor lombar atribuíram o sintoma ao trabalho realizado. $O$ alto nível de intensidade da dor e o grau de incapacidade funcional demonstrou que a tarefa desenvolvida se mostra danosa à saúde dos carregadores. As fortes correlações estatísticas corroboram estas inferências.

A ELN sugeriu uma redução drástica do peso médio da carga de $44,3 \mathrm{~kg}$ para $8,2 \mathrm{~kg}$. O IL médio de 6,0 corrobora a sintomatologia dolorosa reportada pelos carregadores, uma vez que o valor ideal se encontra abaixo de 1,0. Esse resultado indica que a elevação da carga pode ter comprometido a estrutura da coluna lombar dos carregadores, pois manuseavam cargas com aproximadamente $440 \%$ acima do LPR. Não por acaso, em alguns casos a compressão intradiscal superou os limites da literatura, quando os movimentos foram realizados com a carga longe do eixo longitudinal do corpo.

Todavia, este estudo apresenta algumas limitações. As variáveis da tarefa estabelecidas podem não ter coberto a totalidade dos aspectos inerentes às tarefas de levantamento e transporte manual de cargas, devido às distintas condições de trabalho dos carregadores. Além disso, foram contempladas apenas as duas principais metodologias de avaliação de sobrecarga na coluna lombar, não a totalidade existente na literatura. Outro fator que se apresenta como uma limitação do estudo é seu caráter transversal. Dessa maneira, os resultados obtidos referem-se ao período analisado e devem ser generalizados apenas para os participantes que laborem em condições semelhantes aos deste estudo. Assim sendo, pesquisas futuras podem ser realizadas levando-se em consideração as limitações referidas.

De modo geral, as limitações aqui descritas não eliminam as contribuições oferecidas pelo estudo. Sob o ponto de vista teórico, o estudo possibilitou o preenchimento da lacuna existente na literatura com relação a estudos das condições de trabalho de carregadores em centrais de abastecimento no contexto brasileiro. Sob o ponto de vista prático, investigou os principais fatores de riscos de lombalgias existentes nas tarefas executadas por estes profissionais e, sobretudo, evidenciou a necessidade de uma fiscalização mais rígida por parte dos órgãos competentes e que soluções ergonômicas devem ser implementadas imediatamente. 


\title{
Evaluation of the mechanical overload in the lumbar spine of horticultural products loaders: a case study
}

\begin{abstract}
The objective of this study was to evaluate the existence of mechanical overload in the lumbar spine of horticultural loaders of the Central de Abastecimento do Estado de Sergipe (CEASA/SE). For this purpose, data on the variables associated with the handling of loads performed by 60 loaders and the characteristics of self-reported low back pain was collected. Pain intensity and degree of functional disability were evaluated by the Numerical Visual Scale (NVS) and Roland-Morris Disability Questionnaire (RMDQ), respectively. The mechanical overload in the lumbar spine and the risk of developing low back pain were evaluated by the NIOSH Lifting Equation (NLE) and Three-Dimensional Static Strength and Posture Prediction Program (3DSSPP). The results showed that the loaders had a level of pain intensity and a significant degree of functional disability. In addition, it was observed that the loaders handled loads with heavier than recommended weight which consequently caused static multi-directional compression forces on L4/L5 and $L 5 / S 1$ discs of the spine exceeding the limits stipulated in the literature. Thus, the strong correlations between the analyzed variables demonstrated that the task developed is harmful to the health of the loaders.
\end{abstract}

KEYWORDS: Mechanical overload. Load handling. Loader. Low back pain. 


\section{REFERÊNCIAS}

ADAMS, M. A.; HUTTON, W. C. The effect of posture on the role of the apophysial joints in resisting intervertebral compressive forces. The Jurnal of Bone and Joint Surgery, [s.I.], v. 62, $n^{\circ} 3$, p. 358-362, 1980. crossref

ALENCAR, M. C. B. Fatores de risco das lombalgias ocupacionais: o caso de mecânicos de manutenção e produção. 114 p. - Dissertação (Mestrado), Programa de Pós-Graduação em Engenharia de Produção, Universidade Federal de Santa Catarina, UFSC, 2001.

ASSUCEAJU. Relatório de Gestão do Exercício. 2018.

BOGDUK, N. Clinical and Radiological Anatomy of the Lumbar Spine. 5 ed. China: Churchill Livingstone, 2012. 272 p.

BRASIL. PORTARIA MTPS N 3.751, De 23 de novembro de 1990. Norma Regulamentadora 17 - Ergonomia. 1990. Disponível em:

<http://trabalho.gov.br/images/Documentos/SST/NR/NR17.pdf>. Acesso em: 09/mar./18.

BYRNS, G. E. et al. A new direction in low-back pain research. American Industrial Hygiene Association Journal, [s.l.], v. 63, $n^{\circ} 1$, p. 55-61, 2002. crossref

DREISCHARF, M. et al. Estimation of loads on human lumbar spine: A review of in vivo and computational model studies. Journal of Biomechanics, [s.I.], v. 49, $n^{\circ}$ 6, p. 833-845, 2016. ISSN: 00219290. crossref

GORDON, S. J. et al. Mechanism of disc rupture: a preliminary report. Spine, [s.I.], v. $16, n^{\circ} 1$, p. 450-456, 1991. crossref

HJERMSTAD, M. J. et al. Studies comparing numerical rating scales, verbal rating scales, and visual analogue scales for assessment of pain intensity in adults: A systematic literature review. Journal of Pain and Symptom Management, [s.I.], v. $41, n^{\circ} 6$, p. 1073-1093, 2011. crossref

JÄGER, M. et al. Evaluation and assessment of lumbar load during total shifts for occupational manual materials handling jobs within the Dortmund Lumbar Load Study - DOLLY. International Journal of Industrial Ergonomics, [s.I.], v. 25, $\mathrm{n}^{\circ}$ 6, p. 553-571, 2000. ISSN: 01698141. crossref 
MALHOTRA, N. Introdução à pesquisa de marketing. São Paulo: Prentice Hall, 2005.

MÁSCULO, F. S.; VIDAL, M. C. Ergonomia: Trabalho Adequado e Eficiente. 1 ed. Rio de Janeiro: Elsevier Brasil, 2013.

MERINO, E. A. D. Efeitos agudos e crônicos causados pelo manuseio e movimentação de cargas no trabalhador. 128 p. - Dissertação (Mestrado) Universidade Federal de Santa Catarina, Programa de Pós-Graduação em Engenharia de Produção, UFSC, 1996.

MONTEIRO, L. F. et al. Biomechanical Analysis of Manual Baggage Transport Activity in a Brazilian Airport. In: AREZES, P. M. et al. (Orgs.). Proceedings of the International Symposium on Occupational Safety and Hygiene. Guimarães: Portuguese Society for Occupational Safety and Hygiene, 2016. p. 209-211.

MONTEIRO, L. F. et al. Measurement of physical overload in the lumbar spine of baggage handlers at a Brazilian airport. In: AREZES, P. M. et al. (Orgs.). Occupational Safety and Hygiene V. [s.I.]: CRC Press, 2017. crossref

PELLENZ, C. C. O. Indicadores de levantamento de carga e parâmetros mecânicos da coluna vertebral. 130 p. - Dissertação (Mestrado), Programa de Pós-Graduação em Engenharia, Mecânica Universidade Federal do Paraná, UFPR, 2005.

ROLAND, M.; MORRIS, R. A study of natural history of low back pain. Part I: development of reliable and sensitive measure of disability in low back pain. Spine, [s.l.], v. 8, n² 2, p. 141-144, 1983. crossref

SANTA MARIA, A. S. L. Aspectos metodológicos para a determinação de cargas internas na coluna vertebral. - Dissertação (Mestrado em Bioengenharia), Programa de Pós-Graduação em Bioengenharia, Universidade de São Paulo, USP, 2001.

SANTOS, J. W.; MONTEIRO, L. F. Avaliação das condições de trabalho de agentes de bagagem e operadores de rampa de um Aeroporto Brasileiro. Revista Gestão Industrial, [s.I.], v. $13, \mathrm{n}^{\circ} 1,2017$. ISSN: 1808-0448. cross ref

Prospeç̧ão tecnológica sobre acessórios para a estabilização da coluna lombar durante o transporte manual de cargas. Revista Brasileira de Gestão e Inovação, [s.I.], v. 5, $n^{\circ}$ 3, p. 172-194, 2018. crossref

SCHOBER, P.; BOER, C.; SCHWARTE, L. A. Correlation Coefficients: appropriate 
Use and Interpretation. Anesthesia \& Analgesia, [s.I.], v. 126, n 5, p. 1763-1768, 2018. crossref

SILVA, G. V. Movimentação manual de cargas: segurança e saúde dos carregadores autônomos da CEAGESP. 121 p. - Dissertação (Mestrado em Engenharia de Segurança), Universidade Estácio de Sá, Pós-Graduação em Engenharia de Segurança do Trabalho, 2009.

SIMAB. Informações do mercado hortigranjeiro. CONAB. 2019. Disponível em: <http://dw.ceasa.gov.br/>. Acesso em: 14/mar./19.

TEIXEIRA, E. R.; OKIMOTO, M. L. R.; GONTIJO, L. A. Índice de levantamento da equação do NIOSH e lombalgia. Revista Produção Online, [s.I.], v. 11, n 3, p. 735, 2011. ISSN: 16761901 . cross ref

VIOLANTE, F. S.; MATTIOLI, S.; BONFIGLIOLI, R. Low-back pain. Occupational Neurology. [s.I.]: Elsevier, 2015. p. 397-410. crossref

WATERS, T. R. et al. Revised NIOSH equation for the design and evaluation of manual lifting tasks. Ergonomics, [s.I.], v. 36, nº 7, p. 749-776, 1993. ISSN: 00140139. crossref

SANTOS, J.W. et al. Avaliação de sobrecarga mecânica na coluna lombar de carregadores de produtos hortícolas: um estudo de caso. R. Gest. Industr., Ponta Grossa, v. 16, n. 3, p. 247-264, Jul./Set. 2020. Disponível em: https://periodicos.utfpr.edu.br/revistagi

Correspondência:

José Wendel dos Santos

Rua Verde Rosa, número 94, Bairro Lamarão, Aracaju, Sergipe, Brasil.

Direito autoral: Este artigo está licenciado sob os termos da Licença Creative Commons-Atribuição 4.0 Internacional.

\section{(c) (i)}

